\section{Acute renal failure: update}

Robert J. Byrick MD FRCPC
Acute renal failure (ARF) is an abrupt decline in renal function, sufficient to result in the retention of nitrogenous wastes, that is not immediately reversible by manipulation of extrarenal factors. Acute renal failure is not defined in terms of urine volume, and may be present both in oliguric and non-oliguric states. The obligatory daily osmolar load (urea, uric acid, ammonia, etc.) excreted is $400-500 \mathrm{mOsm}$. This represents the normal products of metabolic activity. When urine volume is less than $400 \mathrm{ml}$ per 24 hours, normal osmolar clearance is impossible and nitrogenous wastes accumulate. Such a patient is in oliguric renal failure (ORF). Non-oliguric failure (NORF) occurs when the urine output is excessive for the physiologic state of the patient.

\section{Monitoring renal function}

Urine output, then, is clearly not an optimal index of renal functional status. Urea and creatinine are endogenous products of metabolism excreted primarily by filtration. The blood levels of these products reflect glomerular filtration rate (GFR) if production is constant (doubling of blood level reflects a halving of GFR). With complete cessation of renal function, serum creatinine will rise at a rate of $100-200 \mu \mathrm{g} \cdot \mathrm{L}^{-1} \cdot \mathrm{day}^{-1}$. In an acutely oliguric patient a low urinary sodium concentration $(<20$ $\mathrm{mEq} \cdot \mathrm{L}^{-1}$ ) or a low fractional excretion of sodium calculated (less than one per cent) suggests renal hypoperfusion rather than intrinsic renal damage. ${ }^{1}$ In spite of limitations, the most readily available intraoperative test for diagnosis and monitoring of an oliguric patient remains urine volume. The anaesthetist should establish the basis for oliguria prior to diuretic administration.

\section{Prognosis and causes of acute renal failure}

The clinical setting of ARF involves anaesthetists at many levels. One review ${ }^{2}$ suggested that 43 per cent of cases were related to surgery, 9 per cent to trauma, 14 per cent to pregnancy, 26 per cent occurred in the medical setting, and 8 per cent were directly related to nephrotoxins. Early recognition and treatment of causative factors can reverse the pathophysiology and reduce mortality. Despite aggressive treatment, including dialysis, approximately 60 per cent of patients who develop acute tubular necrosis after surgery and trauma die. ${ }^{2}$ Recognition of predisposing factors leading particularly to post ischaemic acute tubular necrosis is the key to reducing mortality, and the anaesthetist may play a vital role in prevention as we care for patients in the high-risk peri-operative period.

Causes of ARF may be briefly summarized as follows:

\section{Pre-renal}

Pre-renal factors refer to underperfusion of the kidney caused by:

1 Extracellular fluid volume contraction

2 Circulating volume deficit.

3 Reduced cardiac output.

4 Renal vascular disease

Pre-renal dysfunction represents a state of hypoperfusion which, if allowed to persist, leads to structural damage and a parenchymal lesion.

\section{Parenchymal}

Parenchymal causes of ARF refers to intrinsic renal disease and can be classified by the anatomic segment involved.

1 Glomerular diseases.

2 Tubulointerstitial diseases.

3 Vascular disease.

Parenchymal processes may involve nephrotoxins (including fluorinated anaesthetic agents), immunological processes, infectious diseases or ischaemia. Ischaemic factors are of most interest to anaesthetists, who can prevent or ameliorate hypoperfusion states which cause the commonest form of postoperative ARF - acute tubular necrosis or vasomotor nephropathy.

Department of Anaesthesia, St. Michael's Hospital, 30 Bond Street, Toronto, Ontario M5B IW8. 


\section{Post-renal obstruction}

Obstruction due to stones, tumour, etc. may be the sole cause of acute renal failure only if the lesion is bilateral or the patient had only one functioning kidney.

\section{Pathogenesis of ARF}

The pathophysiology of ARF appears to be multifactorial. Factors responsible for causing the renal injury (initiation phase) may be different from those mechanisms that maintain renal dysfunction until recovery (maintenance phase). Therapy based on the pathogenesis of ARF permits an approach to preservation of renal function in the perioperative period. For the anaesthetist, the pathophysiology of acute tubular necrosis is most important and will be emphasized.

\section{Vasomotor response}

Total renal blood flow is reduced $50-75$ per cent in patients with ARF. Renal vasoconstriction causing a critical decrease in glomerular capillary hydrostatic pressure and reduced filtration is observed in patients with ARF regardless of aetiology. Animal models,${ }^{4}$ which often lack the co-existing hypotension, hypoxaemia, sepsis and cardiac failure of patients, also demonstrated preferential reduction in cortical renal blood flow during the initiation phase of ARF. Although this ischaemia may be important in initiating the renal lesion, restoration of cortical flow is not followed by increased GFR once RF is established. Activation of the reninangiotension mechanism that is seen in patients with vasoconstriction has been suspected ${ }^{4}$ as the cause of the low-flow high-resistance state of pre-glomerular arterioles. However, at this time, no convincing evidence exists that angiotensin II blockers (saralasin) or converting enzyme inhibitors (captopril) prevent this vasomotor response to ischaemia.

Other factors such as swelling of the endothelial cells leading to small vessel occlusion, microembolization, increased sympathetic tone, lack of vasodilators $\left(\mathrm{PGE}_{2}\right)$ and decreased activity of kallikrein-kinin system have been inconclusively related to the initiation of the vasomotor response.

Decreased glomerular membrane permeability The factors responsible for maintaining renal failure, however, are not the same as those initiating the insult. A reduced ultrafiltration coefficient of the glomerular membrane has been documented in an ischaemic ARF model. ${ }^{4}$ A 20 per cent decrease in ultrafiltration coefficient was measured within ten hours of injury, eventually dropping to 60 per cent of control values by 48 hours. ${ }^{4}$ Glomerular structural abnormalities in these animal studies have been variable, and may be specific to the norepinephrine infusion model used. The relevance of this factor in the maintenance phase of human ARF is unknown.

\section{Increased filtrate reabsorption}

Backleak of filtrate across damaged tubular epithelium does occur in experimental ARF. With backleak, nearly 100 per cent reabsorption of filtrate would have to occur to cause oliguria. Whether this phenomenon is more important in nephrotoxic than in ischaemic injury is not known.

\section{Tubular blockade}

Increasing evidence suggests that tubular blockade plays a major role in the maintenance phase of ARF in both patients and experimental models. ${ }^{5}$ When low urine flow states exist, urinary viscosity increases and tubular stasis with blockade results. Myoglobin can mechanically obstruct tubules after crush injuries or rhabdomyolysis. Haemoglobin (after transfusion reactions) and plasma proteins, even though poorly filtered, may also obstruct tubules in low flow states.

Tubular casts and debris are found in the urine of patients with ischaemic ATN. Dilated tubules and tubular hypertension can be measured using micropuncture techniques ${ }^{6}$ suggesting tubular obstruction.

Although the histologic term "acute tubular necrosis" is often used synonymously with ARF, actual necrosis of tubular cells may be present in only 10-20 per cent of patients with clinical ARF. There is often little correlation between histologic tubular damage and functional impairment.

\section{Prevention}

Since reversal of ischaemia does not always restore filtration, recognizing clinical states in which ARF is imminent is most important because prompt action by the anaesthetist may prevent the vasomotor response which often initiates ARF. High risk groups are listed in the table: 
TABLE Risk factors for acute renal failure

Clinical shock states

Underlying renal disease

Sepsis

Biliary obstruction (jaundice)

Cirrhosis

Multiple system trauma

Cardiac failure

Extracellular fluid volume contraction

Advanced age

Aorto-renal yascular disease

Invariably, measures to optimize haemodynamic status are essential. The replacement and maintenance of intravascular volume to optimize cardiac output will promote a brisk saline diuresis in most patients, and should precede major operative procedures. The diuresis must be maintained throughout both the procedure and the high-risk postoperative period to minimize tubular obstruction. If such a state of enhanced distal tubular sodium delivery cannot be maintained with intravenous fluids alone, then potent loop diuretics and/or mannitol infusions are warranted

Studies ${ }^{7,8}$ employing furosemide, low-dose dopamine $\left(1-3 \mu \mathrm{g} \cdot \mathrm{kg}^{-1} \cdot \mathrm{min}^{-1}\right)$, or mannitol suggest these agents may help as prophylaxis against potential nephrotoxic or ischaemic insults that cause ATN (vasomotor nephropathy).

Caution is imperative in the administration of diuretics for prophylaxis or treatment of ARF since inadvertent intravascular volume depletion may aggravate renal failure. Conversely, hypertonic mannitol may lead to pulmonary congestion if renal failure delays urinary excretion. Non-oliguric renal failure is increasingly detected and has approximately half the mortality rate of ORF (26 vs. 50 per cent). Non-oliguric forms more frequently follow toxic than ischaemic insults, hence the lower mortality rate may reflect the different cause of ARF or the extent of renal injury. This may also explain why investigators have failed to reduce mortality by "converting" oliguric to non-oliguric ATN with diuretics.

Administration of calcium channel blockers and infusion of prostacyclin have been suggested as prophylactic measures. No convincing evidence of a prophylactic effect in ischaemic clinical situations has been found. Potential nephrotoxic substances, such as aminoglycoside antibiotics, must be used with caution in patients with reduced GFR, such as the elderly or those prone to low flow states.

\section{Anaesthesia and surgery: influence of renal function}

Intraoperative renal function is influenced by multiple factors which are involved in the maintenance of circulating blood volume and ECF composition. Early studies did not monitor circulating intravascular volume as an independent variable and results were confusing. Anaesthesia has little effect on anti-diuretic hormone levels; however, the stress of surgery causes levels to rise, as does cardiopulmonary bypass. ${ }^{9}$ Plasma renin activity (PRA) is important in maintaining cardiovascular stability and intraoperative PRA changes are largely dependent on blood volume and hydration status. ${ }^{9}$

Halothane anaesthesia alone does not impair auto-regulation of renal blood flow. ${ }^{10}$ In ECF volume-expanded patients undergoing aortic reconstructive surgery ${ }^{11}$ minimal changes in GFR are noted after induction of anaesthesia, suggesting that intraoperative and postoperative GFR can be maintained in spite of such factors as intermittant positive pressure ventilation, increased intraabdominal pressure and infra-renal aortic crossclamping, all of which have previously been associated with reduced renal function. Gelman ${ }^{12}$ suggests that during supra-renal aortic cross-clamping, renal blood flow is critically decreased shifting from the cortex to the juxtamedullary layer of the kidney. Vasodilators should be used cautiously in suprarenal cross-clamping ${ }^{12}$ to maintain flow below the aortic occlusion which is pressure dependent. Vasodilators may improve intrarenal blood flow distribution during infra-renal aortic clamping, ${ }^{13}$ as cardiac output and renal blood flow are maintained.

Mannitol, low-dose dopamine infusions and furosemide have been used as prophylactic measures to enhance distal tubular sodium delivery prior to aortic clamping. Our experience ${ }^{11}$ suggests that GFR can be maintained adequately with aggressive intraoperative volume replacement and haemodynamic monitoring for elective infra-renal aortic procedures. For high-risk patients, such as those having supra-renal clamping or emergency procedures, prophylactic mannitol, dopamine or furosemide infusions may be indicated. The protective effects of a drug-induced diuresis result from enhanced distal tubular sodium delivery as well as 
washing debris from tubules, preventing obstruction, and perhaps by preventing pre-glomerular vasoconstriction which initiates the development of ATN.

\section{References}

1 Goldstein MB. Acute renal failure. Med Clin North Am 1983; 67: 1325-41.

2 Mazze RI. Critical care of the patient with acute renal failure. Anesthesiology 1977; 47: 138-48.

3 Hollenberg NK, Epstein M, Rosen SM, Basch RI, Oken DE, Merrill JP. Acute oliguric renal failure in man: evidence for preferential renal cortical ischemia. Medicine 1968; 47: 455-74.

4 Hermreck AS. The pathophysiology of acute renal failure. Am J Surg 1982; 144: 605-10.

5 Stein JH, Lifschitz MD, Barnes LD. Current concepts on the pathophysiology of acute renal failure Am J Physiol 1979; 234: 171-81.

6 Tanner GA, Sophasan S. Kidney pressures after temporary renal artery occlusion in the rat. Am J Physiol 1976; 230: 1173-81.

7 Lindner A, Cutler RE, Goodman WG. Synergism of dopamine plus furosemide in preventing acute renal failure in the dog. Kidney Int 1979; 16: 158-66.

8 Burke TJ, Cronin RE. Duchin KL, Peterson LN, Schrier $R W$. Ischemia and tubule obstruction during acute renal failure in dogs: mannitol in protection. Am J Physiol 1980; 238: F305-F314.

9 Tonneson A. Renal regulatory mechanisms during anesthesia and surgery. American Society of Anesthesiologists Annual Refresher Course Lectures 234: 1985.

10 Bastron RD, Perkins FM. Autoregulation of renal blood flow during halothane anesthesia. Anesthesiology 1977; 46: 142-4.

11 Mazer CD, Rose DK, Goldstein M, Paul M, Byrick $R J$. Renal function in man during infra-renal aortic clamping: influence of mannitol and dopamine. Anesthesiology 1984; 81 : A65.

12 Gelman S, Reves JG, Fowler K, Samuelson PN, Lell $W A, S$ mith $L R$. Regional blood flow during crossclamping of the thoracic aorta and infusion of sodium nitropnusside. J Thorac Cardiovasc Surg 1983; 85: 287-91.

13 Gelman S, Patel K, Bishop SP, Fowler KL, Smith $L R$. Renal splanchnic circulation during infrarenal aortic cross clamping. Arch Surg 1984; 119: 13949. 